Die Schweiz und die humanitäre Aktion

\title{
7. Aussenwirtschaftspolitik
}

\section{Gérard Perroulaz}

\section{(2) OpenEdition}

\section{Journals}

Electronic version

URL: http://journals.openedition.org/sjep/665

DOI: $10.4000 /$ sjep.665

ISSN: 1663-9677

\section{Publisher}

Institut de hautes études internationales et du développement

\section{Printed version}

Date of publication: 1 janvier 1999

Number of pages: $205-226$

ISSN: $1660-5926$

\section{Electronic reference}

Gérard Perroulaz, «7. Aussenwirtschaftspolitik», Schweizerisches Jahrbuch für Entwicklungspolitik [Online], 18 | 1999, Online erschienen am: 28 August 2012, abgerufen am 07 September 2020. URL http://journals.openedition.org/sjep/665 ; DOI : https://doi.org/10.4000/sjep.665 


\section{AUSSENWIRTSCHAFTSPOLITIK*}

D IESES KAPITEL bietet einen Überblick über einige der Instrumente, die in der schweizerischen Aussenwirtschaftspolitik zur Anwendung kommen (ERG, Zollpräferenzen), sowie über die bilateralen Beziehungen der Schweiz mit einigen Entwicklungsländern und mittel- und osteuropäischen Staaten (bilaterale Handelsabkommen, Staatsbesuche, von Mitgliedern des Bundesrates angeführte Delegationen der Schweiz). Die politischen Beziehungen der Schweiz zu den multinationalen Organisationen werden in den Kapiteln über die betreffenden Organisationen behandelt (IWF, Weltbank, UNCTAD, WTO, regionale Entwicklungsbanken).

\subsection{INSTRUMENTE DER SCHWEIZERISCHEN AUSSENWIRTSCHAFTSPOLITIK}

In der Aussenwirtschaftspolitik kommt eine Vielzahl von Massnahmen zum Tragen, die von der Eidgenossenschaft zur Pflege der Aussenhandelsbeziehungen und zum Schutz der weltweiten Interessen der schweizerischen Exporteure und Investoren ergriffen werden. Dank ihrem mehr als 300 Personen zählenden diplomatischen Korps, den in verschiedenen Partnerstaaten tätigen Handelskammern, der Gesamtheit der bilateralen Verträge, der Teilnahme an wichtigen internationalen Konferenzen und der Tätigkeit im Rahmen multilateraler Organisationen, von denen vor allem die WTO in den letzten Jahren immer mehr Bedeutung erlangt hat, verfügt die Schweiz über eine breite Palette von Möglichkeiten zur Vertretung ihrer wirtschaftlichen Interessen. Zur Wahrung der Interessen der Schweizer Investoren im Ausland misst der Bund den bilateralen Investitionsschutz- und Investitionsförderungsabkommen (bislang konnten 86 solche Abkommen abgeschlossen werden) sowie den Doppelbesteuerungsabkommen grosses Gewicht bei. Handelsabkommen wurden vor allem mit den wichtigsten Handelspartnern der Schweiz, aber auch mit einem Teil der aufstrebenden Volkswirtschaften sowie mit potentiellen neuen Handelspartnern abgeschlossen. Die Tabelle 1.1.C.2 des Statistischen Teils gibt einen Überblick über die wichtigsten Handelspartner der Schweiz unter den Entwicklungsländern. Im Güterverkehr mit diesen Ländern weist die Handelsbilanz der Schweiz häufig einen beachtlichen Überschuss aus (in der Regel exportiert die Schweiz mehr Güter nach diesen Ländern, als sie von dort importiert). Die schweizerischen Direktinvestitionen konzentrieren sich auf einige der am weitesten fortgeschrittenen Entwicklungsländer. 1996 entfiel der Grossteil der Direktinvestitionen auf Brasilien, Hongkong, Mexiko, Chile und Singapur.

Die Verantwortung für Angelegenheiten im Bereich der Aussenwirtschaftspolitik obliegt dem Bundesamt für Aussenwirtschaft (BAWI), welches dem Eidgenössischen Volkswirtschaftsdepartement untersteht. Aufgabe des BAWI ist es, den Zugang zu den Märkten zu erleichten und die wirtschaftliche Integration zu

* Von Gérard Perroulaz, Forschungsbeauftragter am IUED, Genf. 
fördern, aber auch im Bereich der Exportförderung tätig zu werden und die Verbesserung der Rahmenbedingungen für die Wettbewerbsfähigkeit des Wirtschaftsplatzes Schweiz voranzutreiben. Ferner ist das BAWI auf dem Gebiet der Zusammenarbeit mit den Entwicklungsländern und den Staaten Mittel- und Osteuropas aktiv (siehe Kapitel 8, Teil 8.4., sowie Kapitel 9 in «Jahresübersicht»). Im Mai 1998 wurde vom Bundesrat die Fusion zwischen dem BAWI und dem Bundesamt für Wirtschaft und Arbeit (vormals BIGA) beschlossen, die es erlauben sollte, sämtliche Kompetenzen im wirtschaftlichen Bereich unter einem Dach zu vereinen und in Zukunft die Interessen der Aussenwirtschaftsund der nationalen Wirtschaftspolitik gleichermassen in die Entscheidungsprozesse einfliessen zu lassen. Der Aufgabenbereich des neugegründeten Bundesamtes, welches seine Arbeit im Jahr 2000 aufnehmen wird, umfasst die Entwicklungszusammenarbeit und die Hilfeleistung gegenüber den osteuropäischen Ländern, die allgemeine Wirtschaftspolitik, die Förderung des Wirtschaftsstandorts Schweiz, die Arbeitsmarktpolitik sowie die Bereiche Arbeitslosenversicherung und Arbeitnehmerschutz.

\subsection{EXPORTRISIKOGARANTIE (ERG)}

Die Exportrisikogarantie (ERG) dient dazu, den Abschluss von Auslandsgeschäften in den Ländern zu erleichtern, wo der Exporteur zögern würde, das Risiko, nicht bezahlt zu werden, auf sich zu nehmen. Die neue Verordnung über die ERG ist am 1. Juli 1998 in Kraft getreten. Die Gebühren (Versicherungsprämien) wurden zur Harmonisierung mit dem zwischen den OECD-Ländern abgeschlossenen Exportkreditarrangement angepasst. Die neue Verordnung setzt die Gebühren nach einer Risikoskala von 1 bis 7 fest, wobei die Abnehmerländer dem Risiko entsprechend in eine der sieben Kategorien eingestuft werden. Ende 1997 erreichte das Gesamtengagement der ERG 6,4 Milliarden Franken, während sich die Neugarantien 1997 auf 2,5 Milliarden Franken beliefen. Der Mittlere Osten und die übrigen Länder Asiens machen über $61 \%$ des Gesamtengagements aus.

\section{$\square$ Allgemeine Funktionsweise der ERG}

Die ERG erlaubt es Schweizer Exporteuren, sich gegen die Nichtzahlung von Lieferungen zu schützen. Der Bund stellt dem schweizerischen Exporteur dieses Instrument zur Verfügung, um ihm den Abschluss von Kaufverträgen im Ausland zu erleichtern. Die ERG deckt die Lieferung oder Vermietung von Waren, die Herstellung von Werken, die Durchführung von Bauarbeiten, die Überlassung von Lizenzen auf immaterielle Güter sowie wissenschaftliche, technische oder wirtschaftliche Beratungsdienste. Garantienehmer sind somit nicht nur der Warenexporteur, sondern auch der Hersteller von Werken oder die Ingenieurfirma. Diese Versicherung wird vom Exporteur sehr geschätzt, wenn wegen der politisch und wirtschaftlich unsicheren Verhältnisse im Abnehmerland Schwierigkeiten beim Zahlungseingang zu befürchten sind. 


\section{Von der ERG gedeckte Risiken}

Die ERG nimmt dem Exporteur bestimmte Risiken bei seinen Auslandsgeschäften ab, die weder von ihm noch vom ausländischen Käufer beeinflussbar sind. Es handelt sich dabei um folgende Risiken:

- Politisches Risiko: Politische Ereignisse (Krieg, Revolution, Aufruhr usw.), die den Abnehmer an der Leistung der vereinbarten Zahlungen hindern oder zum Verlust der noch dem Exporteur gehörenden Ware führen.

- Transferrisiko: Die Zahlungen des Abnehmers werden wegen staatlicher Massnahmen zur Devisenkontrolle oder zur Umschuldung der externen Schuld des Landes nicht überwiesen.

口 Fabrikationsrisiko: Dieses Risiko ergibt sich, wenn eine Ware infolge politischer Ereignisse oder staatlicher Massnahmen nicht geliefert wird.

- Delkredererisiko (kommerzielles Risiko): Zahlungsunfähigkeit oder Zahlungsverweigerung öffentlich-rechtlicher Abnehmer oder gemeinnütziger Unternehmen. Auf Anfrage des Exporteurs kann dieses Risiko auch für private Abnehmer gedeckt werden, wenn diese die Zahlungsgarantie einer von der ERG akzeptierten Bank oder vom Staat erhalten haben.

- Eventualrisiko bei Fremdwährungsgeschäften: Der Exporteur kann sich gegen Zahlung eines Prämienzuschlags die Entschädigung in Schweizer Franken zum Wechselkurs im Zeitpunkt des Schadeneintritts berechnen lassen. Es handelt sich dabei nicht um eine Garantie gegen Wechselkursschwankungen, sondern diese Deckung garantiert lediglich, dass der Exporteur nicht wegen Wechselkursschwankungen unterversichert ist.

Die ERG deckt nicht das Zahlungsrisiko eines privaten Käufers, desgleichen nicht die Risiken, die bei privaten Versicherungsgesellschaften abgedeckt werden können (zum Beispiel Transportrisiken), ferner Währungsrisiken oder Wechselkursschwankungen. Die Währungsrisikogarantie musste 1985 wegen der grossen Verluste, die sie verursacht hatte, abgeschafft werden.

Quelle: Geschäftsstelle für die Exportrisikogarantie, Zürich.

Eine ERG-Kommission aus acht vom Bundesrat ernannten Mitgliedern prüft die Garantiegesuche, die von den Schweizer Exporteuren bei der ERG-Geschäftsstelle in Zürich eingereicht werden ${ }^{1}$. Aufgrund der Vorschläge der Kommission beschliesst das BAWI über die Erteilung der ERG, wenn der Betrag 5 Millionen Franken nicht übersteigt. Beträge über 5 Millionen Franken oder politisch heikle Garantien müssen vom Bundesrat genehmigt werden. Garantien über 10 Millionen Franken müssen ausserdem mit der Zustimmung des eidgenössischen Finanzdepartements gewährt werden.

Das Jahresergebnis der ERG ist seit einigen Jahren positiv, was es der ERG erlaubte, dem Bund seit 1995 einen Teil des Darlehens zurückzuzahlen, das er ihr für ihren Betrieb gewährt hatte. 22 Millionen Franken Zinsen wurden auf diese Weise an den Bund gezahlt, die Schuld wurde um 250 Millionen Franken reduziert (der Bundesvorschuss beläuft sich noch auf 1,34 Milliarden Franken). Zwar weisen die ERG-Geschäfte in den letzten Jahren einen Gewinn aus, jedoch ist zu vermerken, dass die Jahresrechnung über die letzten 24 Jahre in dreizehn Jahren mit einem Verlust (in neun Jahren mit einem Gewinn und in zwei Jahren mit einem ausgeglichenen Saldo) abgeschlossen hat. Die von der

1. Die Kommission bestand 1998 aus vier Vertretern des Bundes (BAWI, DEZA, Finanzverwaltung, Bundesamt für Wirtschaft und Arbeit (BWA), früher BIGA), drei Vertretern der Privatwirtschaft (Schweizerischer Handels- und Industrie-Verein - Vorort, Verein Schweizerischer Maschinen-Industrieller, Schweizerische Gesellschaft für Chemische Industrie) und einer Gewerkschaftsvertreterin (Christliche Gewerkschaft für Industrie, Handel und Gewerbe). Die Kommission wird von Botschafter Rolf M. Jeker (BAWI) geleitet. 
ERG an Exporteure mit Schadenfällen ausgezahlten Entschädigungen beliefen sich 1997 auf 53 Millionen Franken (1996: 108 Millionen Franken).

\section{Neue Verordnung über die ERG}

Im Rahmen der OECD wurde ein Abkommen geschlossen, um die Prämien der staatlichen Exportversicherer zu harmonisieren. Die Gebühr entspricht dem Preis, den der Exporteur zu zahlen hat, um durch die Exportrisikogarantie gedeckt zu sein (Versicherungsprämie). Das Exportkreditarrangement wurde von den Ministern der OECD-Staaten am 20. Juni 1997 angenommen. Es regelt die Mindestgebühren für staatliche Exportkreditgarantien mit einer Laufzeit von über zwei Jahren. Die Gebühren beruhen auf einer gemeinsamen Risikoeinstufung der Abnehmerländer mit einer Skala von 1 bis 7. Die Einstufung wird im Einvernehmen zwischen den Sachverständigen der OECD-Mitgliedsstaaten anhand der makroökonomischen Daten des betreffenden Landes sowie der Erfahrungen der verschiedenen Versicherer hinsichtlich der Zahlungen periodisch revidiert. Die Länder müssen somit eine Mindestgebühr anwenden.

Die Schweiz hat sich für das OECD-Arrangement ausgesprochen, das eine bessere Disziplin unter den Ländern ermöglicht. Der Wettbewerb zwischen den Unternehmen wird dadurch auf technischen Kriterien und wettbewerbsfähigen Preisen statt auf Unterschieden in den Exportsubventionssystemen beruhen. Die Anwendung einer von Land zu Land verschieden hohen Gebühr zieht in der Tat eine Verzerrung des Wettbewerbs nach sich, so dass ein Unternehmen eines Landes eine höhere staatliche Unterstützung erhält als ein Unternehmen eines anderen Landes (die Wettbewerbsverzerrungen können sogar zwischen dem Unternehmen eines Landes und einer Tochterfirma des gleichen Unternehmens im Ausland bestehen).

Das OECD-Arrangement tritt nach Ablauf einer Übergangsfrist zur Anpassung der nationalen ERG-Systeme am 1. April 1999 in Kraft. In der Schweiz ist die neue ERG-Verordnung mit der Gebührenrevision am 1. Juli 1998 in Kraft getreten $^{2}$. Die neue schweizerische Verordnung setzt somit die Grundgebühren fest, die sich entsprechend der Garantiedauer und den sieben Länderrisikogruppen erhöhen. Zum Beispiel muss ein Unternehmen, dessen Ausfuhren (bei einer Garantiedauer von fünf Jahren) zu einer Million Franken versichert sind, eine Grundgebühr zwischen 8500 Franken und 74'000 Franken zahlen, je nachdem, ob das Abnehmerland als wenig oder sehr gefährlich gilt. Zusätzliche Gebühren können für Risiken erhoben werden, die nicht durch die Grundversicherung gedeckt sind (zum Beispiel Einschluss des Währungsrisikos ${ }^{3}$ ). Bei vermindertem Risiko können auch Gebührenermässigungen gewährt werden (zum Beispiel bei Beteiligung internationaler Finanzierungsinstitutionen oder bei Lieferungen im Rahmen der bilateralen Finanzhilfe, nichtstaatlicher Anteil der Mischfinanzierungen beispielsweise).

\section{$\square$ Neugarantien 1997}

Wie aus der linken Spalte von Tabelle 1 ersichtlich, belief sich der Gesamtbetrag der 1997 erteilten Neugarantien auf 2,5 Milliarden Franken, was einer Stei-

2. «Verordnung über die Exportrisikogarantie» vom 15. Juni 1998, Bern, in der Amtlichen Sammlung des Bundesrechts, Nr. 26, 7. Juli 1998, S. 1624-1642. Diese Verordnung ersetzt die Verordnung vom 15. Januar 1969.

3. Seit Juli 1996 kann der Exporteur sich gegen Zahlung eines Prämienzuschlags die Entschädigung in Schweizer Franken zum Wechselkurs im Zeitpunkt des Schadeneintritts berechnen lassen. 
gerung von $74 \%$ gegenüber dem Vorjahr (1,4 Milliarden) entspricht. $87 \%$ der Neugarantien wurden an folgende Länder (in sinkender Grössenordnung der Beträge) vergeben: Türkei (über 700 Millionen Franken Neugarantien), China (über 400 Millionen), Bahrain, Iran, Saudiarabien, Mexiko, Marokko, Israel, Polen und Brasilien. 83\% der Neugarantien entfielen auf die Maschinenindustrie, 14\% auf die chemische Industrie. Die sechs grössten Projekte (von je 50 Millionen Franken) machten 39\% des Neugarantievolumens aus.

\section{Drei-Schluchten-Damm in China}

Eines der grössten Projekte, für das von der ERG 1997 eine Garantie (zugunsten der Firma Asea Brown Boveri, ABB) vergeben wurde, betrifft die Materialausfuhr für den Drei-Schluchten-Damm in China. ABB hat einen Auftrag über vierzehn Generatoren für einen Betrag von 340 Millionen Franken erhalten. Die Kampagnen der Erklärung von Bern und der Arbeitsgemeinschaft der Hilfswerke hatten sich 1996 und 1997 auf den Widerstand gegen die ERG-Vergabe für die schweizerischen Lieferungen beim Bau des Drei-Schluchten-Damms konzentriert. 25'000 von der Erklärung von Bern vorbereitete Postkarten wurden an den Bundesrat und ABB mit der Aufforderung versandt, auf die ERGErteilung für dieses umstrittene Grossstaudammprojekt zu verzichten. Ferner forderten die Kampagnen eine Reform der Funktionsweise der ERG (Berücksichtigung der Ziele nachhaltiger Entwicklung und der Menschenrechte bei der Gewährung der ERG, Erweiterung der ERG-Kommission auf eine/n Vertreter/in der Hilfswerke, bessere Transparenz bei den erteilten Garantien).

[] JSDW 1998, S. 282-284, Einzelheiten der Kampagne.

\section{Ilisu-Staudamm in der Türkei}

Im November 1998 haben sich die Erklärung von Bern, der WWF Schweiz und die Hilfswerke an den Bundesrat mit der Aufforderung gewandt, ABB und Sulzer die Exportrisikogarantie zur Deckung der Ausfuhren für den Bau von Bewässerungsanlagen am Euphrat und Tigris in der Türkei zu verweigern (es handelt sich dabei um den Ilisu-Staudamm im Rahmen des GAP-Projekts in Südostanatolien). Die beiden Schweizer Firmen haben eine Garantie für einen Betrag von 470 Millionen Franken beantragt. Mehrere Argumente wurden von den NRO angeführt, um die Ablehnung der ERG-Erteilung für dieses Grossprojekt zu verlangen, das als nicht übereinstimmend mit einer kohärenteren Politik der Schweiz gegenüber den Entwicklungsländern beurteilt wird. Zum ersten würde das Projekt die politischen und sozialen Gegebenheiten in einem Kurdengebiet der Türkei verändern. Die Umsiedlung der Bevölkerung könnte den Zustrom von Menschen in die Slums der türkischen Grossstädte und den Strom kurdischer Flüchtlinge in die Schweiz verstärken. 15'000 bis 20'000 Personen müssten durch das Projekt umgesiedelt werden, und die Erlangung von Entschädigungen und angemessenen Bedingungen bei der Umsiedlung der Bevölkerung sei bei einem als repressiv bekannten Regime nicht gesichert. Zum zweiten würde die Schweiz durch die Gewährung der ERG die von den Nachbarländern im Süden (Irak und Syrien) als aggressiv erachtete türkische Politik indirekt unterstützen. Mit dem riesigen Reservepotential des Stausees des Kraftwerks könnte das GAPProjekt in der Tat eine Bedrohung für die Wasserversorgung dieser am Unterlauf der Flüsse Euphrat und Tigris gelegenen Länder darstellen. Zudem habe sich die Türkei der UN-Konvention über den nichtschiffbaren Gebrauch internationaler Wasserwege nicht angeschlossen, welche grenzüberschreitende Konflikte bei der 
Wassernutzung regeln sollte. Der dritte Punkt betrifft die Auswirkungen des Stauwerks auf die Umwelt und die Gesundheit (Verbreitung von Malaria). Ferner halte das Projekt die Richtlinien der Weltbank nicht ein, die das Projekt im übrigen nicht finanziell unterstützt. Schliesslich stelle der Bau auch eine Bedrohung für bestimmte archäologische Stätten und Kulturgüter dar.

Ende November 1998 hat der Bundesrat beschlossen, die Erteilung der ERG für Aufträge im Wert von 470 Millionen Franken für den Ilisu-Staudamm (und von 285 Millionen Franken für das Gaswerk Ankara) zu bewilligen. Der Bundesrat begründet den positiven Entscheid für das Ilisu-Projekt mit der Anerkennung des Energiebedarfs der Türkei, mit der Bedeutung der Projekte für die Beschäftigung in der Schweiz und mit gewissen Garantien, die von der Türkei bezüglich der Entschädigung der umzusiedelnden Bevölkerung gegeben wurden. Die ERG-Kommission hat die Meinung von Nichtregierungsorganisationen angehört, bevor sie die Annahme des Projekts empfahl. Erstmals fanden auch Konsultationen mit dem Ziel einer internationalen Koordination zwischen den sieben Exportgarantiestellen der anderen Länder statt, wo von Exportunternehmen Garantieanträge gestellt worden waren. Der Bundesrat hat das Wirtschaftsdepartement beauftragt, im Einvernehmen mit den übrigen betroffenen Ländern einen Dialog mit den für den Bau des Staudamms Verantwortlichen zu führen und einen unabhängigen Kontrollmechanismus (Monitoring) einzurichten, um sicherzustellen, dass die Umsiedlung der Bevölkerung nach international akzeptablen Standards erfolgt.

Tabelle Nr. 15: Geographische Aufteilung der Neugarantien und des Gesamtengagements der ERG 1997

\begin{tabular}{lrrrr}
\hline & \multicolumn{2}{c}{$\begin{array}{c}\text { Neugarantien } \\
\text { 1997 }\end{array}$} & \multicolumn{2}{c}{$\begin{array}{c}\text { Gesamtengagement } \\
\text { per 31.12.1997 }\end{array}$} \\
\hline Ländergruppen & $\begin{array}{r}\text { Millionen } \\
\text { Franken }\end{array}$ & $\begin{array}{r}\text { Anteil } \\
\text { in \% }\end{array}$ & $\begin{array}{r}\text { Millionen } \\
\text { Franken }\end{array}$ & $\begin{array}{r}\text { Anteil } \\
\text { in \% }\end{array}$ \\
\hline Europa (ohne Mittel- $u$. Osteuropa) & 755.6 & 30.5 & 1128.8 & 17.6 \\
Mittel- und Osteuropa & 118.5 & 4.8 & 195.3 & 3.0 \\
\hline Zentral- und Südamerika & 164.7 & 6.6 & 646.9 & 10.1 \\
\hline Afrika & 147.9 & 6.0 & 509.9 & 7.9 \\
\hline Mittlerer Osten & 713.5 & 28.8 & 1544.1 & 24.0 \\
\hline Übriges Asien & 579.4 & 23.4 & 2382.8 & 37.1 \\
\hline Australien & 0.0 & 0.0 & 12.9 & 0.2 \\
\hline Total & $\mathbf{2 4 7 9 . 6}$ & $\mathbf{1 0 0 . 0}$ & $\mathbf{6 4 2 0 . 7}$ & $\mathbf{1 0 0 . 0}$ \\
\hline davon: 70 ärmere Entwicklungsländer & 550.7 & 22.2 & 1186.3 & 118.5 \\
\hline \multicolumn{1}{c}{ davon: Amerika } & 0.0 & 0.0 & 2.5 & 0.0 \\
\hline \multicolumn{1}{c}{ Afrika } & 18.1 & 0.7 & 125.2 & 1.9 \\
\hline \multicolumn{1}{c}{ Asien } & 532.6 & 21.5 & 1058.6 & 16.5 \\
\hline
\end{tabular}

Quelle: ERG-Jahresbericht 1997, Juni 1998, Tabelle Anhang C/1.

\section{$\square$ Gesamtengagement der ERG}

Ende 1997 belief sich das Gesamtengagement der ERG auf 6,4 Milliarden Franken (rechte Spalte von Tabelle 15). Die Graphik 4 führt die fünfzehn wichtigsten ERG-gedeckten Länder auf. Diese Länder machen 83,7\% des gesamten ERG-Engagements aus. Indonesien, die Türkei, China und der Iran entsprechen zusammen $53 \%$ aller Verpflichtungen. 
Das Gesamtengagement hat sich von Ende 1996 bis Ende 1997 für die Türkei stark erhöht (von 531 Millionen Franken Ende 1996 auf über eine Milliarde Franken Ende 1997), desgleichen für China (von 372 Millionen auf 680 Millionen Franken) und für Bahrain (von 49 Millionen auf 214 Millionen Franken). Mit den Neuverpflichtungen der ERG für 1998 hat die Türkei sogar seit dem Frühjahr 1998 Indonesien überholt und ist unter den fünfzehn wichtigsten ERGgedeckten Ländern an die erste Stelle gerückt.

Die geographische Aufteilung des ERG-Engagements weist folgende Hauptmerkmale auf (Stand Ende 1997):

\section{Europa}

Die europäischen Länder machen 17,6\% des ERG-Gesamtengagements, das heisst über 1,1 Milliarden Franken aus. Diese Verpflichtungen entfallen jedoch grösstenteils auf die Türkei (über 1 Milliarde Franken).

\section{Mittel- und Osteuropa}

Das ERG-Engagement in diese Region beläuft sich lediglich auf 195 Millionen Franken, das heisst 3\% des Gesamtengagements. Diese Verpflichtungen dienen hauptsächlich dazu, Ausfuhren nach Polen (67 Millionen Franken Ende 1997) und nach Rumänien (64 Millionen) zu decken. Jedoch ist zu vermerken, dass es ein Instrument zusätzlicher Garantien aus den Rahmenkrediten für die mittelund osteuropäischen Länder gibt. Diese Garantien werden für Länder gewährt, für welche die ERG wegen eines als zu hoch eingeschätzten Risikos nicht (oder nur teilweise) zur Verfügung steht (vgl. Kapitel 9 betreffend die Instrumente der öffentlichen Hilfe für diese Länder). Das Engagement gegenüber Russland ist ziemlich gering (6 Millionen Franken Ende 1997).

\section{Mittlerer Osten}

Der Mittlere Osten macht ein Viertel des Gesamtengagements aus, vor allem für Ausfuhren in den Iran, nach Saudiarabien, Israel, Bahrain und Jordanien.

\section{Übriges Asien}

Der relative Anteil Asiens ist hoch (37\% der Gesamtgarantien, plus 24\%, wenn man die Länder des Mittleren Ostens mitzählt).

Unter den von der Wirtschafts- und Finanzkrise betroffenen asiatischen Ländern ist die ERG sehr stark in Indonesien engagiert (mit 1,1 Milliarden Franken) und in erheblich geringerem Masse in den Philippinen (116 Millionen Franken), in Thailand (69 Millionen) und in Südkorea (4,5 Millionen). In Japan und Malaysia hat die ERG kein Engagement. Infolge der Krise und des Vertrauensschwunds bei einigen Exporteuren können bei der ERG künftig möglicherweise vermehrt Anträge für Geschäfte in diesen Ländern eingehen (besonders für Geschäfte, die bislang ohne Exportrisikogarantie finanziert wurden, da sie als wenig riskant galten). Die ERG hat im Sommer 1998 erklärt, sie bleibe angesichts der Zahlungsunfähigkeitsrisiken bei den umfangreichen ERG-gedeckten Exporten nach Indonesien weiterhin gelassen (namentlich wegen der hohen Rückstellungen, die von der ERG für Notfälle vorgesehen wurden).

\section{Mittel- und Südamerika}

Auf Mittel- und Südamerika entfallen 10\% des Gesamtengagements (647 Millionen Franken), vor allem zur Deckung von Ausfuhren nach Brasilien (214 Millionen Franken), Mexiko (176 Millionen), Argentinien (101 Millionen) und Kolumbien (60 Millionen). 


\section{Afrika}

Lediglich 8\% der ERG-Garantien für schweizerische Exporte betreffen den afrikanischen Kontinent (510 Millionen Franken). Die wichtigsten ERG-gedeckten Länder sind Algerien (104 Millionen Franken), Ägypten (99 Millionen), Marokko (93 Millionen), Südafrika (65 Millionen) und Simbabwe (45 Millionen).

\section{Graphik Nr. 4: Die 15 wichtigsten ERG-gedeckten Länder Gesamtengagement per 31. Dezember 1997, in Millionen Franken}

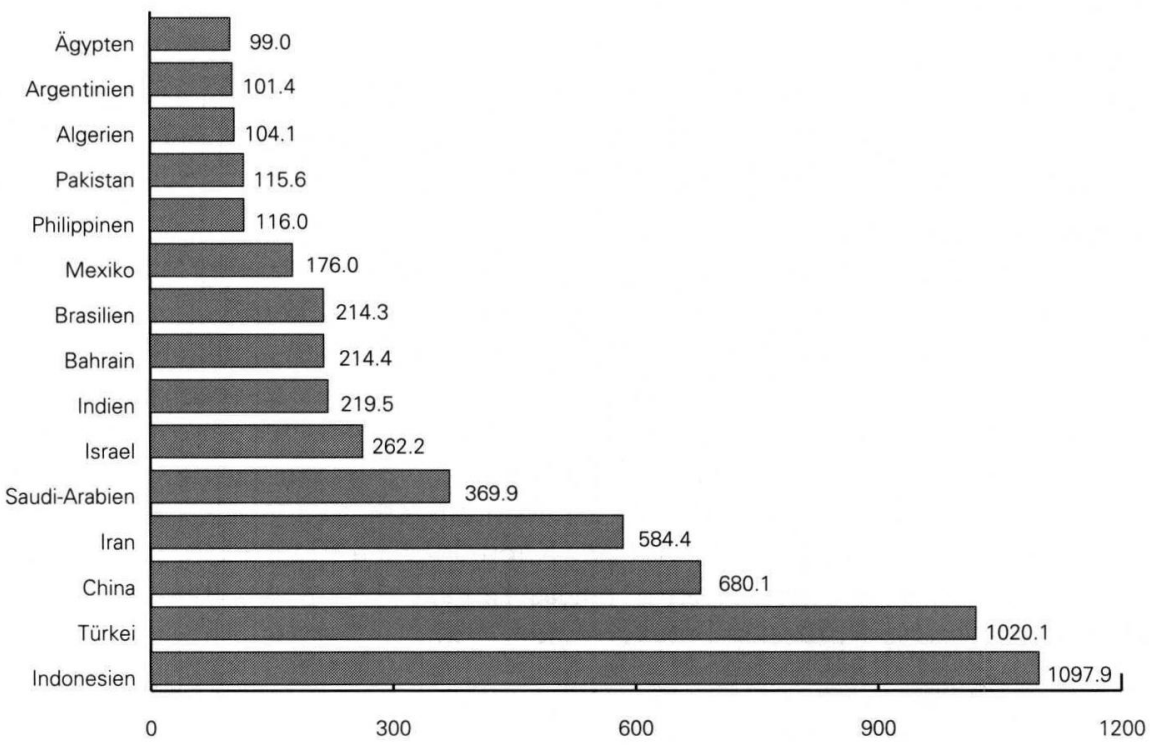

Quelle: ERG-Jahresbericht 1997, Juni 1998, anhand der Tabellen von Anhang G/1 bis G/3 angefertigte Graphik.

\section{$\square$ Ärmere Entwicklungsländer}

1980 hatte das Parlament dem ERG-Gesetz folgende Bestimmung hinzugefügt (Art. 1, Abs. 2): «Bei Exporten nach ärmeren Entwicklungsländern hat der Bund die Grundsätze der schweizerischen Entwicklungspolitik mitzuberücksichtigen.» Die vom Bund festgelegte Liste der 70 ärmeren Entwicklungsländer umfasst die 48 am wenigsten entwickelten Länder (LDC) sowie 22 weitere einkommensschwache Länder ${ }^{4}$ (Länder mit einem BSP pro Einwohner unter 765 Dollar 1996). Zwischen 1996 und 1997 wurden Albanien und Georgien von der Liste der ärmeren Länder gestrichen.

1997 entfielen 96,7\% der Neugarantien für die ärmeren Länder auf den asiatischen Kontinent. Das Gesamtengagement der ERG für diese Staaten erreichte 1997 1186,3 Millionen Franken, was 18,5\% des Gesamtengagements für alle Länder entspricht. $89 \%$ der gesamten Verpflichtungen für diese Ländergruppe entfielen auf Asien, 10,6\% auf Afrika.

4. Die Liste der 70 ärmeren Länder umfasst die 48 am wenigsten entwickelten Länder (LDC) sowie folgende Länder: Armenien, Aserbeidschan, Bosnien, China, Elfenbeinküste, Ghana, Guyana, Honduras, Indien, Kamerun, Kenia, Kirgistan, Kongo, Mongolei, Nicaragua, Nigeria, Pakistan, Senegal, Simbabwe, Sri Lanka, Tadschikistan und Vietnam. 


\section{$\square$ Schuldenkonsolidierungsabkommen}

Ende 1997 waren 90 bilaterale Umschuldungsabkommen mit 31 Ländern in Kraft. Die aus diesen Abkommen erwachsenden ERG-Guthaben erreichten 3,1 Milliarden Franken (inklusive kapitalisierte Zinsen und angefallene, aber nicht bezahlte Zinsen). Diese Entschuldungsmassnahmen erlauben es der ERG, die konsolidierten Forderungen an den Bund abzutreten.

1997 wurden vom Bund sechs neue bilaterale Umschuldungsabkommen mit fünf Ländern abgeschlossen: Kamerun, Guinea-Conakry (mit zwei Abkommen), Jordanien, Madagaskar und Russland. Der gesamte Umschuldungsbetrag beläuft sich auf 425,5 Millionen Franken. Der ERG-Jahresbericht stellt fest, dass sich die Zahlungsbereitschaft und die Zahlungsfähigkeit der Schuldnerländer in den letzten Jahren verbessert. So wurden 1997 81\% der in Rechnung gestellten Kapitalrückzahlungen (1996: 82\%) und 95\% der geforderten Zinsen (1996: 84\%) effektiv beglichen.

JSDW 1999, Kapitel 1.2., der «Jahresübersicht» über die internationalen Finanzbeziehungen, den Abschnitt über die Entschuldungsmassnahmen der Schweiz.)

\section{QUELLEN}

ERG-Geschäftsstelle und BAWI, ERG Jahresbericht1997, Zürich und Bern, Juni 1998.

«Verordnung über die Exportrisikogarantie» vom 15. Juni 1998, Bern (Amtliche Sammlung des Bundesrechts, Nr. 26, 7. Juli 1998, S.1624-1642).

Eidgenössisches Wirtschaftsdepartement, Pressemitteilung vom 22. Juni 1998, Presse- und Informationsdienst, Bern. Le Temps, 23. Juni 1998.

Neue Zürcher Zeitung, 23. Juni 1998, «Noch keine Grippesymptome bei der ERG», 25. November 1998, «Umstrittene ERG für Kraftwerk in der Türkei», 1. Dezember 1998, «Exportrisikogarantie für türkische Kraftwerke».

Tribune de Genève, 25. November 1998, «llisu, un projet de barrage turc qui embarasse la Suisse».

Erklärung von Bern, Internet-Adresse: http://www.access.ch.evb/db

ERG-ADRESSEN

Geschäftsstelle für die Exportrisikogarantie, Kirchenweg 8, 8032 Zürich.

INTERNET-ADRESSE

http://www.swiss-erg.com/

\subsection{ZOLLPRÄFERENZEN}

Seit 1972 gewährt die Schweiz Präferenzzollansätze für die Einfuhr bestimmter Güter aus Entwicklungsländern. Im Rahmen der UNCTAD wurden auf multilateraler Ebene Zielsetzungen und allgemein geltende Prinzipien für die Handhabung der Zollpräferenzen vereinbart, nach denen die nationalen Präferenzschemen periodisch überprüft werden. Im September 1996 wurde von den Eidgenössischen Räten eine Revision des Präferenzschemas beschlossen. Die neue Zollpräferenzverordnung, in der die neuen Präferenzzollansätze festgelegt sind, trat am 1. März 1997 in Kraft. Der Inhalt dieser neuen Verordnung sowie die Handhabung der Präferenzen zwischen 1994 und 1996 wurde in der Ausgabe 1998 des Jahrbuchs diskutiert (siehe JSDW 1998, S. 286-291). Von den wichtigsten Neuerungen, die mit dem neuen Präferenzschema eingeführt wurden, wäre der «Grundsatz der Vollgraduierung» zu nennen, wonach der Entwicklungsstand des Partnerlandes ausschlaggebend für die Gewährung von Präferenzzöllen ist. Gemäss diesem Grundsatz kann die Schweiz die Zollpräferenzen zugunsten der am weitesten fortgeschrittenen Entwicklungsländer aufheben. Mit Wirkung auf den 1. März 1998 hat die Schweiz denn auch die 
Präferenzzollansätze für Einfuhren aus verschiedenen Ländern (darunter Südkorea, Hongkong, Mexiko und Singapur) aufgehoben. Ferner hat die Schweiz aufgrund des bereits seit längerem bestehenden «Grundsatzes der Teilgraduierung» die Möglichkeit, nur für bestimmte Waren, die aus einem Land importiert werden, die Zollpräferenzen aufzuheben. So werden beispielsweise für Textilimporte aus China und Macao keine Präferenzzollansätze mehr gewährt.

\section{$\square$ Zollpräferenzen 1995-1997}

Die folgende Tabelle weist die Beträge für die präferenzzollbegünstigten Importe der vergangenen Jahre aus. Rund drei Viertel der Einfuhren aus Entwicklungsländern könnten durch Präferenzzölle begünstigt werden (dies entspricht ca. 6-8\% der schweizerischen Gesamtimporte). Tatsächlich aber liegt der Ausnützungsgrad der Präferenzen relativ niedrig: Nur knapp die Hälfte der verfügbaren Zollpräferenzen wird effektiv in Anspruch genommen (47\% im Jahr 1997). Während der Ausnützungsgrad für Agrarprodukte sehr hoch ist, liegt er für Industrieprodukte relativ tief, da die Präferenzmarge für gewisse industrielle Erzeugnisse sehr gering ist und die Exporteure aus diesem Grund davon absehen, die notwendigen Schritte zu unternehmen, um in den Genuss der Zollpräferenzen zu kommen. Tatsächlich wurden 1997 im Rahmen der Zollpräferenzen Güter im Wert von 2,6 Milliarden Franken eingeführt, darunter fallen auch Importe in der Höhe von 340 Millionen Franken, auf die keinerlei Zölle erhoben wurden.

Tabelle Nr. 16: Schweizerische Einfuhren und Zollpräferenzen 1995-1997

\begin{tabular}{lccr}
\hline Einfuhren und Präferenzen & $\mathbf{1 9 9 5}$ & $\mathbf{1 9 9 6}$ & $\mathbf{1 9 9 7}$ \\
\hline $\begin{array}{l}\text { 1. Gesamtvolumen der schweizerischen } \\
\quad \text { Einfuhren in Mio. Fr. }\end{array}$ & 94483 & 96664 & 110087 \\
\hline $\begin{array}{l}\text { Einfuhren aus Entwicklungsländern } \\
\text { in Mio. Fr. }\end{array}$ & 6175 & 5828 & 8318 \\
\hline $\begin{array}{l}\text { Anteil der Einfuhren aus Entwicklungsländern } \\
\text { in \% (Punkt 2 in \% von Punkt 1) }\end{array}$ & $6.5 \%$ & $6.0 \%$ & $7.6 \%$ \\
\hline $\begin{array}{l}\text { Einfuhren, für die Präferenzen gewährt werden } \\
\text { können, in Mio. Fr. }\end{array}$ & 4223 & 4326 & 5633 \\
\hline $\begin{array}{l}\text { Tatsächlich ausgenutzte Präferenzen } \\
\text { in Mio. Fr. }\end{array}$ & 2188 & 2204 & 2649 \\
\hline $\begin{array}{l}\text { Ausnützungsgrad der gewährten Präferenzen } \\
\text { in \% (Punkt 5 in \% von Punkt 4) }\end{array}$ & $52 \%$ & $51 \%$ & $47 \%$ \\
\hline
\end{tabular}

Quelle: BAWI

\subsection{EXPORTFÖRDERUNG}

Der Bund leistet finanzielle Beiträge an im Bereich der Exportförderung tätige Organisationen wie z.B. die schweizerischen Handelskammern im Ausland. Die Schweizer Zentrale für Handelsförderung (Office suisse d'expansion commerciale, OSEC), eine offizielle Institution der Schweiz zur Förderung des Aussenhandels, deren Budget zu einem Drittel durch den Bundeshaushalt finanziert wird, wurde vom Bund 1997 mit fast 10 Millionen Franken unterstützt. Aufgabe des OSEC ist es, die Aussenhandelsbeziehungen zu fördern und das Image der Schweizer Wirtschaft weltweit zu pflegen. Ferner unterstützt das OSEC aber 
auch die Exportwirtschaft bei der Suche nach neuen Absatzmärkten im Ausland, bietet Beratungs- und Informationsdienstleistungen über die Absatzmärkte und die schweizerische Exportwirtschaft an und ist regelmässig an Handelsmessen vertreten ${ }^{5}$.

Zur Zeit wird im Bundesrat ein neues Gesetz über die Förderung des Aussenhandels vorbereitet. Der Grundgedanke des neuen Gesetzes ist es, die Voraussetzungen für den Abschluss eines Leistungsvertrags zwischen dem Bund und einem Managementunternehmen zu schaffen. So könnte mit den Unterstützungszahlungen, die bisher an das OSEC, an die Handelskammern oder an die Berufsverbände ausgerichtet wurden, ein Managementunternehmen entschädigt werden, das auf der Grundlage von vom BAWI ausgearbeiteten Zielvorgaben tätig wird. Auf diese Weise könnte die finanzielle Unterstützung besser an die tatsächlichen Erfolge gebunden werden, die im Bereich der Exportförderung erzielt wurden ${ }^{6}$.

\subsection{BILATERALE WIRTSCHAFTSABKOMMEN}

\section{$\square$ Investitionsschutz- und Investitionsförderungsabkommen}

Die Schweiz hat im Laufe der Zeit ein sehr dichtes Netz von bilateralen Abkommen zum Schutz und zur Förderung von Investitionen errichtet. Ein Investitionsschutzabkommen deckt sämtliche Kategorien von Eigentum in einem bestimmten Land ab: Besitz von Gütern und Immobilien, Aktien, verschiedenste Formen von Unternehmensbeteiligungen, Konzessionen, Urheberrechte und gewerbliche Eigentumsrechte (Patente, gewerbliche Muster und Modelle, Marken- und Handelsnamen). Beide Vertragsparteien verpflichten sich, im Rahmen des Möglichen die Investitionstätigkeit des Partnerstaats zu fördern. Dabei müssen allerdings die Grundsätze der Meistbegünstigung und der Nichtdiskriminierung respektiert werden, was bedeutet, dass ein Partnerstaat die Investitionen aus den anderen Staat nicht strenger behandeln darf als Investitionen aus einem Drittstaat (diesbezügliche Ausnahmen sind selten und kommen nur vor, wenn der Staat z.B. einer Freihandelszone angehört). Die freie Verfügung über die Erträge aus den Investitionen (Zinsen, Dividenden, zusätzliche Kapitalzufuhren zur Förderung der Investitionen, Erträge aus einer teilweisen oder vollständigen Liquidierung des Investitionsobjekts) muss ebenfalls gewährleistet sein. Allfällige Enteignungen oder Verstaatlichungen können nur unter Ausrichtung einer adäquaten Entschädigungszahlung vorgenommen werden. Streitigkeiten zwischen den beiden Vertragsstaaten oder zwischen einem Investor und einem Vertragsstaat sind mittels Konsultationen zu regeln und können vor ein Schiedsgericht getragen werden.

Bis Ende November 1998 hat die Schweiz nahezu 90 Investitionsschutzabkommen abgeschlossen. Die Tabelle auf Seite 293 und 294 des Jahrbuchs 1998 gibt eine vollständige Übersicht über die Abkommen, die mit Entwicklungsländern und mittel- und osteuropäischen Staaten abgeschlossen worden sind. 1997 und 1998 traten Abkommen mit Südafrika, Kuba und Kasachstan in Kraft. Abkom-

\footnotetext{
5. OSEC, Av. de l'Avant-Poste 4, 1001 Lausanne. Tel.: 021/320 32 31, Fax 021/320 73 37, Internet-Adresse: http://www.osec.ch.

6. Info@Bawi, Nr. 2, September 1998, BAWI.
} 
men mit den folgenden Staaten wurden in den vergangenen Jahren zwar unterzeichnet, sind aber (Ende November 1998) noch nicht in Kraft getreten: Kambodscha und Zimbabwe (Unterzeichnung der Abkommen 1996), Indien, Mongolei, Philippinen und Thailand (Unterzeichnung der Abkommen 1997), Äthiopien, Armenien, Botswana, Mauritius, Iran, Kuweit, Vereinigte Arabische Emirate (Unterzeichnung der Abkommen 1998). Tabelle 17 dieses Kapitels bietet eine vollständige Liste der in Kraft getretenen Abkommen mit mittel- und osteuropäischen Ländern und GUS-Staaten.

\section{$\square$ Doppelbesteuerungsabkommen}

Die Unterzeichnerstaaten eines Doppelbesteuerungsabkommens verzichten auf einen Teil ihrer Steuerhoheit, um zu verhindern, dass Einkommen doppelt, d.h. in beiden der betroffenen Staaten, besteuert werden. Solche bilateralen Abkommen regeln die Besteuerung von natürlichen Personen und Unternehmen und decken die unterschiedlichsten Einkommensarten ab: Einkommen, Einkünfte aus unbeweglichem Vermögen, Dividenden, Zinsen auf Forderungen, Gebühren, Kapitalgewinne, Einkünfte von Künstlern und Sportlern, Renten. Falls das Vermögen nur in einem der beiden Vertragsstaaten besteuert wird, betrifft das Abkommen nur die Einkommenssteuern. Nach der Unterzeichnung muss ein solches Abkommen vom Parlament gutgeheissen werden, allerdings untersteht es nicht dem fakultativen Referendum.

Im Jahrbuch 1998 sind sämtliche Länder aufgeführt, mit denen die Schweiz ein Doppelbesteuerungsabkommen abgeschlossen hat (JSDW 98, S. 293/294). Bis 1998 hat die Schweiz mehr als 50 derartige Abkommen geschlossen. Erst seit 1996 bestehen auch mit folgenden Ländern Doppelbesteuerungsabkommen: Argentinien, Slowakische Republik, Slowenien (1996), Thailand, Venezuela (unterzeichnet im Dezember 1996, in Kraft seit Anfang 1998), Vietnam (unterzeichnet 1996, in Kraft seit 1. Januar 1998). Mit mehreren Ländern, darunter die Vereinigten Arabischen Emirate, sind die Verhandlungen noch nicht abgeschlossen.

\section{$\square$ Freihandelsabkommen}

Aufgrund der Ausdehnung der Europäischen Union zählt die Europäische Freihandelsvereinigung (EFTA) seit 1995 nur noch vier Mitgliedsstaaten, nämlich Island, Liechtenstein, Norwegen und die Schweiz. Die mit Drittstaaten abgeschlossenen Freihandelsabkommen haben zum Ziel, den in diese Länder exportierenden Unternehmen dieselben Bedingungen zuzusichern, die auch die Unternehmen aus den Ländern der EU geniessen. Die Abkommen decken den Handel mit Industrieprodukten und verarbeiteten landwirtschaftlichen Erzeugnissen, aber auch mit einer beschränkten Anzahl landwirtschaftlicher Produkte ab. Für Agrarprodukte, die nicht in diese Kategorie fallen, werden Zollvergünstigungen gewährt. Die Abkommen enthalten ausserdem Bestimmungen über die

Beilegung von Streitigkeiten sowie über den Schutz des geistigen Eigentums. Die EFTA ist bestrebt, Abkommen mit den südlichen Mittelmeerländern abzuschliessen, dies um so mehr, als die EU mittelfristig die Schaffung einer Freihandelszone zwischen Europa und diesen Ländern vorsieht (die 1995 geschlossene Partnerschaft mit elf Mittelmeeranrainerstaaten). Freihandelsabkommen zwischen der EU und einigen Staaten dieser Region (Israel, Jordanien, Marokko, PLO, Tunesien) sind bereits unter Dach und Fach, Verhandlungen mit weiteren Staaten (Algerien, Ägypten und Libanon) sind im Gange. 
Freihandelsabkommen zwischen der EFTA und den folgenden 13 Ländern sind bereits in Kraft getreten (Stand September 1998): Bulgarien, Estland, Israel, Lettland, Litauen, Marokko, Polen, Rumänien, Slowakei, Slowenien, Tschechien, Türkei und Ungarn. Ferner wurden mit Albanien, Jordanien, Ägypten, Libanon, Mazedonien, der PLO und Tunesien Vereinbarungen zur Zusammenarbeit eingegangen, welche auf den Abschluss von Freihandelsabkommen abzielen (die Verhandlungen mit Tunesien sind schon weit vorangeschritten, während Ägypten dem Abschluss des Abkommens mit der EU als vorrangiges Ziel betrachtet). Ferner sind Verhandlungen zum Abschluss von Freihandelsabkommen mit Malta und Zypern im Gange. Die EFTA strebt zudem ein derartiges Abkommen mit den Mitgliedern des Kooperationsrats der Golfstaaten (Arabische Emirate, Oman und Saudiarabien) an. Die mit den Baltischen Staaten geschlossenen Abkommen wurden 1997 von den Eidgenössischen Räten gebilligt. Bereits in Kraft getretene Verträge werden regelmässig überarbeitet und an die Veränderungen in der internationalen Wirtschaftspolitik angepasst (technische Regelungen, Zugang zum öffentlichen Beschaffungswesen, Schutz des geistigen Eigentums, Dienstleistungen und Investitionen). Revisionen dieser Art wurden kürzlich an den Abkommen mit Bulgarien, Israel, Rumänien und der Türkei vorgenommen.

Am 19. Juni 1997 wurde das Freihandelsabkommen mit Marokko abgeschlossen, welches von der Schweiz 1998 ratifiziert wurde. Es handelt sich dabei um das erste derartige Abkommen mit einem nordafrikanischen Staat. Marokko ist nach Südafrika und Ägypten der wichtigste afrikanische Markt für die schweizerische Exportwirtschaft. Dank diesem Abkommen konnte der Freihandel mit Industrieprodukten, verarbeiteten landwirtschaftlichen Erzeugnissen sowie mit Fischereiprodukten abgedeckt werden. Das Abkommen beinhaltet insofern eine gewisse Asymmetrie, als die EFTA-Staaten ihre Zölle umgehend mit dem Inkrafttreten des Abkommens aufheben, während Marokko eine Übergangsperiode von 12 Jahren zur Aufhebung seiner Schutzzölle zugestanden wurde. Der Landwirtschaftssektor wurde in bilateralen Vereinbarungen zwischen den EFTA-Staaten und Marokko geregelt (Zollsenkungen für gewisse Produkte, welche jedoch nicht über die Zugeständnisse, die den anderen Freihandelspartnern der Schweiz gemacht wurden, hinausgehen).

\section{$\square$ Abkommen über Handel und wirtschaftliche Zusammenarbeit}

Am 10. Mai 1997 konnte zwischen der Schweiz und der Kirgisischen Republik ein Abkommen über Handel und wirtschaftliche Zusammenarbeit unterzeichnet werden. Das Abkommen wurde 1998 von den Eidgenössischen Räten gebilligt und besitzt eine Geltungsdauer von fünf Jahren (die jeweils um fünf Jahre verlängert werden kann). Ziel dieses Abkommens ist die Förderung der Wirtschaftsbeziehungen zwischen den beiden Ländern und die Unterstützung des Reformprozesses zum Übergang zu einer Marktwirtschaft in Kirgisistan. Dabei stützt sich das Abkommen auf die von der WTO festgelegten Grundsätze: Beide Länder verpflichten sich zur gegenseitigen Anwendung der Meistbegünstigungsklausel und zum Verzicht auf diskriminierende Praktiken in den Wirtschaftsbeziehungen. Aus dem Partnerstaat importierte Güter erhalten dieselbe Behandlung wie einheimische Produkte, und der Zahlungsverkehr erfolgt in einer auf dem Devisenmarkt handelbaren Währung (Verzicht auf Tausch- oder Kompensationsgeschäfte). Ferner verpflichten sich die Vertragsstaaten zur 
Bekämpfung der Nachahmungen und Fälschungen sowie zu einem angemessenen Schutz des geistigen Eigentums auf der Grundlage der im Rahmen der WTO geschlossenen Verträge (TRIPS), der Pariser Verbandsübereinkunft zum Schutze des gewerblichen Eigentums, der Berner Übereinkunft zum Schutz von Werken der Literatur und Kunst und dem Rom-Abkommen (Schutz der ausübenden Künstler, Hersteller von Tonträgern und der Sendeunternehmen), wobei eine Übergangsfrist von drei Jahren vereinbart wurde. Die Wirtschaftszusammenarbeit nimmt einen wichtigen Platz ein, sie soll dazu beitragen, den Güteraustausch zu konsolidieren und zu diversifizieren, die Entwicklung der Volkswirtschaft zu unterstützen, die Strukturanpassungen voranzutreiben (Konsolidierung der Kirgisischen Republik im Bereich der Wirtschaftspolitik) und den Anteil der KMU am Güteraustausch zu fördern, sie umfasst aber auch die technische Hilfestellung im Bereich des Schutzes des geistigen Eigentums. Zur Überwachung der Einhaltung des Abkommens wurde eine gemischte Kommission mit Vertretern beider Vertragsparteien eingesetzt.

Das Handelsvolumen zwischen der Schweiz und der Kirgisischen Republik ist sehr gering: Es werden praktisch keine Güter in die Schweiz eingeführt, und die schweizerischen Exporte nach Kirgisistan beliefen sich 1995 auf weniger als eine Million Franken. Der 1996 verzeichnete Anstieg des Handelsvolumens ist vornehmlich auf die Lieferung von Maschinen zurückzuführen, die im Rahmen von Hilfskrediten zugunsten mittel- und osteuropäischer Länder finanziert wurden.

Weitere Abkommen über Handel und wirtschaftliche Zusammenarbeit bestehen bereits mit Mazedonien (in Kraft seit 1996) und Kasachstan (Abschluss 1994, in Kraft seit dem 1. Juli 1997). Ähnliche Abkommen wurden ferner mit Armenien (Unterzeichnung im November 1998), der Russischen Föderation, der Ukraine, Usbekistan, Belarus und Moldau geschlossen. Im Juni 1997 konnte ein weiterer Vertrag mit Georgien paraphiert werden, die Verhandlungen über ein Abkommen mit Turkmenistan sind noch im Gange.

\section{Abkommen mit Vietnam über den Schutz des geistigen Eigentums und die Zusammenarbeit auf diesem Gebiet}

Dank dieses Abkommens, das 1998 vom Parlament gebilligt wurde ${ }^{7}$, ist die Schweiz in der Lage, Vietnam bei der Errichtung eines legalen und institutionellen Rahmens auf dem Gebiet des geistigen Eigentums Hilfe zu leisten. Im Gegensatz zu den anderen Freihandelsverträgen oder bilateralen Abkommen, die in der Regel auch Bestimmungen bezüglich des geistigen Eigentums enthalten, handelt es sich bei diesem Abkommen um das erste seiner Art, das sich ausschliesslich auf den Bereich des geistigen Eigentums bezieht.

Die Vertragsparteien verpflichten sich, einen wirksamen und nichtdiskriminierenden Schutz des geistigen Eigentums zu gewährleisten und die dazu nötigen gesetzlichen Bestimmungen auszuarbeiten. Vietnam, das der WTO noch nicht beigetreten ist, verpflichtet sich im Rahmen dieses Abkommens, ab dem 1. Januar 2000 einen Schutz zu gewähren, der nicht unter den in den Verträgen der Uruguay-Runde (TRIPS) festgelegten Standards liegt und der zum Beispiel

7. «Botschaft bezüglich des Abkommens zwischen der Schweizerischen Eidgenossenschaft und der Sozialistischen Republik Vietnam über den Schutz des geistigen Eigentums und über die Zusammenarbeit auf dem Gebiet des geistigen Eigentums», BB1 Nr. 9, 10. März 1998 (Anhang zum «Bericht zur Aussenwirtschaftspolitik 97/1+2»). 
Urheberrechte, geographische Ursprungsbezeichnungen, gewerbliche Muster und Modelle, Patente und Pflanzensorten umfasst. Gemäss des Grundsatzes der Inländerbehandlung darf ein Schweizer Staatsbürger gegenüber einem vietnamesischen Staatsangehörigen nicht benachteiligt werden, und die Meistbegünstigungsklausel stellt die Gleichberechtigung von Schweizern gegenüber Angehörigen anderer Staaten sicher. Die Schweiz verpflichtet sich im Rahmen dieses Abkommens, während vorerst drei Jahren ein spezielles Zusammenarbeitsprogramm im technischen Bereich durchzuführen, welches Vietnam bei der Verstärkung des Schutzes des geistigen Eigentums unterstützen soll. Das Programm umfasst verschiedene Aspekte, wie z.B. die Anpassung der nationalen Gesetzgebung, die Ausbildung von qualifiziertem Personal sowie die Modernisierung der Infrastruktur im Bereich der Informatik. Das spezielle Zusammenarbeitsprogramm wird aus dem Rahmenkredit für die Weiterführung wirtschaftsund handelspolitischer Massnahmen im Bereich der internationalen Entwicklungszusammenarbeit finanziert und deckt unter anderem folgende Gebiete ab (Auszüge aus Anhang 2 des Abkommens):

- Bestimmung der Bedürfnisse zur Modernisierung der rechtlichen Rahmenbedingungen in Vietnam und Ausarbeitung neuer Gesetzesvorschläge zum Schutz des geistigen Eigentums;

- Qualifizierte Ausbildung der Mitarbeiter der für den Schutz des geistigen Eigentums zuständigen Behörden;

- Modernisierung der Informationssysteme im Patentwesen und Bereitstellung der benötigten technischen Hilfsmittel;

- Sensibilisierung der Öffentlichkeit für die Notwendigkeit, Erfindungen und Schöpfungen zu schützen und Nachahmungen und Fälschungen zu bekämpfen;

- Ermutigung der Unternehmen, lokalen Gemeinschaften und Einzelpersonen, die Rechte zum Schutz des geistigen Eigentums in allen Bereichen der Technologie in Anspruch zu nehmen.

凹SDW 1998, S. 299 im Teil Jahresübersicht, sowie den Artikel von T. Thu-Lang Tran im Teil «Analysen und Stellungnahmen» S. 47-64.

Wirtschaftsabkommen zwischen der Schweiz und den mittelund osteuropäischen Staaten

Die folgende Tabelle bietet einen Überblick über die bilateralen Verträge zwischen der Schweiz und den mittel- und osteuropäischen Ländern und den GUSStaaten. 
Tabelle Nr. 17: Wirtschaftsabkommen zwischen der Schweiz und den Ländern des Ostens (Datum des Inkrafttretens) - Stand September 1998

\begin{tabular}{lcccc}
\hline & $\begin{array}{c}\text { Abkommen über Handel } \\
\text { und wirtschaftliche } \\
\text { Zusammenarbeit }\end{array}$ & $\begin{array}{c}\text { Freihandels- } \\
\text { abkommen }\end{array}$ & $\begin{array}{c}\text { Investitions- } \\
\text { schutzabkommen }\end{array}$ & $\begin{array}{c}\text { Doppel- } \\
\text { besteuerungs- } \\
\text { abkommen }\end{array}$ \\
\hline Albanien & 1996 & - & 1993 & en prép. \\
\hline Bosnien-Herzegowina & - & - & - & - \\
\hline Bulgarien & 1973 & 1994 & 1993 & 1993 \\
\hline Estland & 1926 & 1997 & 1993 & en prép. \\
\hline Kroatien & en prép. & - & 1997 & - \\
\hline Lettland & 1925 & 1997 & 1993 & en prép. \\
\hline Litauen & - & 1997 & 1993 & en prép. \\
\hline Mazedonien & 1996 & - & 1997 & en prép. \\
\hline Polen & 1973 & 1994 & 1990 & 1992 \\
\hline Rumänien & 1973 & 1994 & 1994 & 1994 \\
\hline BR Jugoslawien & - & - & - & - \\
\hline Slowakei & 1971 & 1992 & 1991 & 1997 \\
\hline Slowenien & - & $1995^{* *}$ & 1997 & $1996^{*}$ \\
\hline Tschechien & 1971 & 1992 & 1991 & 1996 \\
\hline Ungarn & 1974 & 1994 & 1989 & 1982 \\
\hline Armenien & en prép. & - & - & - \\
\hline Aserbaidschan & en prép. & - & - & - \\
\hline Georgien & en prép. & - & - & - \\
\hline Kazachstan & 1997 & - & 1998 & 1988 \\
\hline Kirgistan & 1998 & - & en prép. & - \\
\hline Moldau & 1996 & - & 1996 & en prép. \\
\hline Russland & 1995 & - & 1991 & 1997 \\
\hline Tadjikistan & - & - & - & - \\
\hline Turkmenistan & en prép. & - & - & - \\
\hline Ukraine & 1996 & - & 1997 & 1998 \\
\hline Usbekistan & 1994 & - & 1993 & en prép. \\
\hline Belarus & 1994 & - & 1994 & en prép. \\
\hline ren & & - & & \\
\hline
\end{tabular}

Quelle: «Botschaft über die Weiterführung der verstärkten Zusammenarbeit mit Osteuropa und den Staaten der GUS" vom 19. August 1998, Botschaft Nr. 98.049, Anhang II.

* Unterzeichnung

** Vorläufiges Inkrafttreten

\subsection{WIRTSCHAFTSDIPLOMATIE}

Die Besuche der Bundesräte in den Entwicklungsländern bieten vor allem Gelegenheit zur Diskussion wirtschaftlicher Fragen (zu den Delegationen der Schweiz zählen auch Vertreter der Privatwirtschaft). Nicht zuletzt aber werden diese Treffen genutzt, um bilaterale Verträge zu unterzeichnen und diversen Projekten der Entwicklungszusammenarbeit einen Besuch abzustatten. Die vom Bundesrat angeführten Delegationen sollen der Schweizer Privatwirtschaft den Zugang zu den Ministerien der besuchten Länder erleichtern und es ihnen erlauben, Kontakte auf hoher Ebene im Partnerstaat zu knüpfen, indem den Unternehmen Gelegenheit geboten wird, mit Regierungschefs, Aussenministern, Handels- und Industrieministern, Wirtschaftsministern sowie den für die Energiewirtschaft und Telekommunikation zuständigen Regierungsbeamten zusammenzutreffen. 


\section{ASIEN}

\section{Thailand/Malaysia}

Sein erster offizieller Besuch ausserhalb Europas Anfang 1998 führte Bundesrat Pascal Couchepin an der Spitze einer rund 30 Personen zählenden gemischten Delegation, der auch Wirtschaftsvertreter angehörten, nach Thailand und Malaysia. Thailand ist einer der wichtigsten Handelspartner der Schweiz unter den Entwicklungsländern (das Land lag 1996 auf dem vierten Rang der schweizerischen Importe und an achter Stelle der Exportländer). 1997 betrugen die Einfuhren aus diesem Land 560 Millionen Franken, während sich die Ausfuhren nach Thailand auf 901 Millionen Franken beliefen (im Falle von Malaysia betrugen die Einfuhren in die Schweiz im selben Jahr 178 Millionen Franken, die Ausfuhren 628 Millionen Franken). Im ersten Halbjahr 1998 wurde ein Rückgang der schweizerischen Exporte nach diesen Ländern um ein Drittel verzeichnet. Die schweizerischen Investitionen in Malaysia betrugen 1996 insgesamt 858 Millionen Franken. Damit nimmt die Schweiz den fünften Rang unter den ausländischen Investoren ein (die Investitionen in Thailand beliefen sich auf 692 Millionen Franken). Die Gespräche in Thailand drehten sich um die Geldund Finanzkrise, das ambitiöse Privatisierungsprogramm der Regierung sowie die Revision des Patentrechts. Im Oktober 1998 stattete die malaysische Handels- und Industrieministerin Bundesrat Couchepin in Bern einen Gegenbesuch ab. Bei dieser Gelegenheit verlieh Couchepin seiner Besorgnis hinsichtlich der Massnahmen der malaysischen Regierung zur Kontrolle der Wechselkurse und Kapitalflüsse Ausdruck.

\section{Vereinigte Arabische Emirate/Kuweit}

Eine Delegation unter der Leitung von Staatssekretär Franz Blankart bereiste vom 30. Oktober bis zum 6. November 1998 die Vereinigten Arabischen Emirate und Kuweit. Die Delegation zählte unter anderem Vertreter der Uhrenindustrie, der Banken, der Chemiebranche, der Nahrungsmittel- und Maschinenindustrie, des OSEC sowie der Arabisch-Schweizerischen Handels- und Industriekammer. Die Gespräche drehten sich vornehmlich um die Frage der Uhrenfälschungen und um die Nachahmung von Medikamenten schweizerischer Herkunft. Ferner wurden Investitionsschutzabkommen mit Kuweit und den Vereinigten Arabischen Emiraten unterzeichnet. Mit den Emiraten soll ein Doppelbesteuerungsabkommen ausgehandelt werden, und von Schweizer Seite wurde der Wunsch geäussert, ein Freihandelsabkommen zwischen der EFTA und dem Kooperationsrat der Golfstaaten abzuschliessen. Für die schweizerische Exportwirtschaft stellt diese Region einen bedeutenden Absatzmarkt dar: 1997 betrugen die Ausfuhren nach Saudiarabien 1,076 Milliarden Franken, nach den Vereinigten Arabischen Emiraten 610 Millionen, nach Kuweit 212 Millionen, nach Oman 156 Millionen, nach Bahrain 106 Millionen und nach Katar 82 Millionen Franken.

\section{Indien}

Ende November 1998 reiste Bundesrat Flavio Cotti mit einer Delegation von Vertretern der Bundesverwaltung und der schweizerischen Privatwirtschaft nach Indien. Das Volumen des Handels zwischen den beiden Ländern ist beachtlich, und die Schweiz gehört zu den zehn wichtigsten Investoren in Indien. Die schweizerischen Exporte nach Indien beliefen sich 1997 auf 591 Millionen Franken, wovon der grösste Teil auf Werkzeugmaschinen, Apparate, Textil- und Druckereimaschinen, chemische Erzeugnisse und Pharmazeutika entfiel. Im 
gleichen Jahr wurden Güter, hauptsächlich Textilien, im Wert von 451 Millionen Franken aus Indien in die Schweiz importiert. Abgesehen von den Wirtschaftsgesprächen standen Besuche bei einer gewissen Anzahl von Projekten der Zusammenarbeit auf dem Programm (Herstellung FCKW-freier Kühlschränke, Seidenproduktion, Kleinkreditprogramm). Indien ist das wichtigste Empfängerland der Schweizer Entwicklungszusammenarbeit, obgleich die Eidgenossenschaft das Budget für die Zusammenarbeit im Anschluss an die indischen Nuklearversuche um 5\% gekürzt hatte. Im wirtschaftlichen Bereich bestehen mehrere Divergenzen. So wirft die Schweiz der indischen Regierung vor, die von ihr errichteten Hindernisse für die Einfuhr hochwertiger Textilprodukte und Luxusuhren seien $\mathrm{zu}$ hoch. Die indischen Uhrenhersteller beklagen sich ihrerseits über den schwierigen Zugang zu den Handelsmessen in der Schweiz. Hingegen hat die indische Regierung seit Herbst 1998 die Revision des Patentgesetzes vorangetrieben, welches bis 2005 den Anforderungen des im Rahmen der WTO getroffenen TRIPS-Abkommens entsprechen muss (dabei geht es insbesondere um die Ausweitung des Patentschutzes auf Produkte und nicht mehr nur wie bisher auf Herstellungsverfahren) und ausländischen Unternehmen die Möglichkeit geben sollte, ihre Patente anzumelden.

JSDW 1998, Dossier: geistige Eigentumsrechte, S. 25-46 (Artikel von D.M. Nachane «Intellectual property rights and the WTO: A Southern perspective») und S. 97-106 (Artikel von P. S. Mehta» TRIPS and pharmaceuticals: Implications for India»).

\section{Weitere Besuche von Bundesräten oder Wirtschaftsdelegationen}

๖ Während seiner Reise in den Nahen Osten im Mai 1998 führte Bundesrat Flavio Cotti politische Gespräche mit Jordanien und Israel.

๖ Versammlung der gemischten Kommission Schweiz-Usbekistan im März 1998. Zweck des Treffens war ein Gedanken- und Informationsaustausch hinsichtlich der Wirtschaftslage, der weiteren Entwicklung der bilateralen Beziehungen und der Möglichkeiten zur Entwicklung der für Handel und Investitionen nötigen Rahmenbedingungen. Bei den schweizerischen Einfuhren aus Usbekistan handelt es sich fast ausschliesslich um Textilien (6 Millionen Franken 1997). Die Exporte der Schweiz nach Usbekistan sind mit 23 Millionen Franken ebenfalls relativ gering (hauptsächlich Agrarprodukte, Maschinen, chemische Erzeugnisse und Pharmazeutika).

• Eine gemischte Delegation von Vertretern der Maschinen- und Uhrenindustrie, der Ingenieurbüros, der Nahrungsmittelindustrie, des Chemiesektors, des Bankenwesens und der Handelsunternehmen besuchte unter der Leitung von Botschafter Nicolas Imboden im März 1998 den Iran. Anlässlich des Besuchs wurde unter anderem ein Investitionsschutzabkommen unterzeichnet.

\section{LATEINAMERIKA}

\section{Mexiko}

Bundesrat Pascal Couchepin weilte vom 6. bis zum 10. Oktober zu einem Besuch in Mexiko. Er wurde dabei von einer Delegation von rund zwölf Vertretern der Schweizer Wirtschaft begleitet. Anlässlich dieses Besuchs wurde eine gemeinsame Absichtserklärung zur wirtschaftlichen Zusammenarbeit unterzeichnet. Dieses Dokument bildet die Grundlage für ein gemeinsames Arbeitsprogramm auf dem Gebiet der Liberalisierung des Dienstleistungs- und Investitionsbereichs, der Verstärkung der wirtschaftlichen Zusammenarbeit und der 
Förderung der Handels- und Investitionsbeziehungen. Die gemeinsame Erklärung stellt einen ersten Schritt zur Schaffung einer Freihandelszone zwischen der EFTA und Mexiko dar (Mexiko ist im Begriff, mit der Europäischen Union Verhandlungen über ein Freihandelsabkommen aufzunehmen).

Bereits 1993 haben beide Länder ein Doppelbesteuerungsabkommen unterzeichnet, das 1995 von einem Investitionsschutzabkommen gefolgt wurde. Die schweizerischen Exporte nach Mexiko, hauptsächlich Maschinen (38\%), chemische Erzeugnisse (37\%), aber auch Pharmazeutika, Uhren, Instrumente und Apparate, beliefen sich 1997 auf 634 Millionen Franken. Die Einfuhren aus Mexiko setzen sich zum grössten Teil aus chemischen Erzeugnissen, Agrarprodukten (davon 46\% Kaffee und 30\% Honig) sowie Maschinen zusammen und beliefen sich im selben Jahr auf 68 Millionen Franken. Die schweizerischen Investitionen in Mexiko betrugen 1997 1'845 Millionen Franken (kumulierter Wert) ${ }^{8}$. 20'300 Personen sind bei Schweizer Unternehmen in Mexiko beschäftigt. Ausserdem ist Mexiko das zweitwichtigste lateinamerikanische Land für schweizerische Investitionen.

Brasilianisches Staatsoberhaupt in der Schweiz

Fernando Henrique Cardoso weilte im Januar 1998 zu einem Staatsbesuch in der Schweiz. Das 1994 unterzeichnete Investitionsschutzabkommen ist vom brasilianischen Parlament noch nicht ratifiziert worden. Das Forum SchweizBrasilien, eine Plattform schweizerischer NRO, zur Unterstützung von Projekten zur Entwicklungszusammenarbeit in Brasilien, überreichten dem Präsidenten eine Petition, in der sie eine verstärkte Einhaltung der Rechte des Kindes und der autochthonen Völker sowie die Durchführung einer Agrarreform forderten. Brasilien ist der wichtigste Handelspartner der Schweiz in Lateinamerika und das wichtigste Bestimmungsland für schweizerische Investitionen auf diesem Kontinent (Ende 1996 betrugen die schweizerischen Investitionen in Brasilien mehr als 4,9 Milliarden Franken).

\section{AFRIKA}

\section{Südafrika}

Vom 2. bis zum 6. August 1998 weilte Bundesrat Flavio Cotti zu einem Staatsbesuch in Südafrika. Er wurde von einer Wirtschaftsdelegation begleitet, der unter anderem Vertreter der Unternehmen Asea-Brown Boveri (ABB), Ascom, Kuoni, Holderbank und der UBS angehörten. Dies war der vierte Besuch eines Bundesrates in Südafrika seit 1994. Präsident Nelson Mandela hat seinerseits ebenfalls schon viermal die Schweiz besucht.

Südafrika ist der wichtigste Absatzmarkt für schweizerische Exporte auf dem afrikanischen Kontinent (558 Millionen Franken im Jahr 1997). Ferner ist Südafrika nach Nigeria der zweitwichtigste afrikanische Lieferant der Schweiz (die Einfuhren aus Südafrika beliefen sich 1997 auf 395 Millionen Franken). Die Schweiz importiert in erster Linie Rohstoffe, Agrarprodukte und Wein. Hinzuzufügen ist jedoch, dass die Goldimporte in den Zollstatistiken nicht berücksichtigt werden. Südafrika ist weltweit der wichtigste Lieferant dieses Edelmetalls, das zum Teil in der Schweiz raffiniert und weiterverkauft wird. Berücksichtigt man den Handel mit Gold ebenfalls, so nimmt die Schweiz unter 
den Exportmärkten Südafrikas den ersten Rang ein, gefolgt von den USA und Grossbritannien'. Unter den Schweizer Exporten nach Südafrika finden sich hauptsächlich Maschinen (30\% der Exporte), chemische Erzeugnisse und Pharmazeutika (rund 35\%) sowie Lebensmittel.

Unter den ausländischen Investoren in Südafrika nimmt die Schweiz hinter den USA, Deutschland, Grossbritannien, Malaysia und Südkorea den sechsten Platz ein. 300 Schweizer Unternehmen besitzen Niederlassungen in diesem Land, dies entspricht Investitionen in der Höhe von rund 4 Milliarden Franken. Über 25'000 Personen sind bei schweizerischen Unternehmen beschäftigt, beinahe 6900 allein bei Nestlé ${ }^{10}$. Das gegenseitige Investitionschutz- und Investitionsförderungsabkommen, welches 1995 unterzeichnet wurde, ist am 29. November 1997 in Kraft getreten.

\section{Beziehungen zwischen der Schweiz und Südafrika: Überschattete Vergangenheit}

Die Beziehungen zwischen den beiden Ländern wurden in der Vergangenheit nicht zuletzt durch die Tatsache überschattet, dass sich die Schweiz dem von den Vereinten Nationen ausgerufenen internationaIen Boykott des Apartheid-Regimes und den von einigen Staaten (beispielsweise den Vereinigten Staaten und der Europäischen Union) ergriffenen Sanktionen nicht angeschlossen hatte. Zwischen 1986 und 1989 verzeichneten die schweizerischen Importe aus Südafrika einen markanten Zuwachs von 154 Millionen auf 1,178 Milliarden Franken. Ein wichtiger Grund dafür war der Direktimport von Diamanten durch eine Tochtergesellschaft des südafrikanischen Unternehmens De Beers. Zuvor waren die Diamantimporte über Grossbritannien abgewickelt worden und erschienen folglich in den Statistiken als Einfuhren aus jenem Land.

Die Nationalrätin Pia Hollenstein (Grüne/SG) forderte im März 1997 den Bundesrat auf, eine historische Untersuchung über die Beziehungen der Schweiz zum Apartheid-Regime in die Wege zu leiten. In seiner Antwort erklärte der Bundesrat, er sehe keine Notwendigkeit zur Durchführung einer solchen Untersuchung, und lieferte darüber hinaus eine Rechtfertigung der schweizerischen Politik gegenüber Pretoria*. 1986 hatte sich die Schweiz gegen die Ergreifung von Wirtschaftssanktionen ausgesprochen. Sie verteidigte den Grundsatz der Universalität der Wirtschaftsbeziehungen, der einen Eckpfeiler der schweizerischen Aussenpolitik darstellt, verurteilte aber gleichzeitig die in Südafrika praktizierte Politik der Apartheid. In seiner Antwort räumte der Bundesrat allerdings ein, die Schweiz würde heute nicht unbedingt dieselbe Position einnehmen; die damalige Haltung sei zwar verständlich gewesen, zeuge aber nicht von politischem Weitblick.

Eine Plattform schweizerischer NRO ist im Begriff, eine Kampagne über die Geschäftspraktiken Schweizer Unternehmen unter dem Regime der Rassentrennung in Südafrika zu lancieren. Diese Kampagne soll dazu beitragen, Licht auf die Haltung des öffentlichen und des privaten Sektors während dieser Zeit zu werfen und die Schuldverschreibungen des alten Regimes rückgängig zu machen (die NRO erachten die Darlehen und Kredite, die dem Apartheid-Regime zugestanden wurden, als illegitim). Von der Bevölkerung Südafrikas (und der angrenzenden Länder, die unter den Destabilisierungsaktionen des Apartheidregimes gelitten haben) dürfe nicht verlangt werden, dass sie den Preis für die Apartheid mit der Tilgung der Schulden ein zweites Mal bezahlen müsse**. Die von den Unternehmen und den Banken angehäuften Gewinne sind nach Ansicht der NRO als illegitim zu deklarieren, gleichzeitig seien diese Unternehmen zur Wiedergutmachung gegenüber der Bevölkerung verpflichtet.

* Le Temps, 20. Juli 1998: "Rouvrir le dossier Suisse-Afrique du Sud ne répond à aucune nécessité».

** "Aktion Südliches Afrika. Entschädigen-Apartheidschulden streichen». Siehe auch die Homepage der COTMEC (Commission Tiers Monde de l'Eglise catholique), Tel.: 022/329.26.81) http://www.cath.ch/cotmec, Rubrik Aktionen. Weitere Informationen bei: Aktion Finanzplatz Schweiz Dritte Welt, Basel, Tel.: 061/693 17 00. Die Kampagne wird bereits von rund 30 schweizerischen, einer Koalition südafrikanischer und einem Dutzend deutscher NRO unterstützt (Juni 1998).

9. Neue Zürcher Zeitung, 3.9.1997.

10. Quellen der Zahlen: Neue Zürcher Zeitung, 3. August 1998. Die von der Schweizerischen Nationalbank veröffentlichten Zahlen über den Investitionsbestand sprechen von Investitionen im Wert von 1,1 Milliarden Franken, wobei seit 1994 ein Anstieg verzeichnet werden kann. 
Zwischen 1986 und 1994 flossen Hilfsgelder in der Höhe von 50 Millionen Franken von der Schweiz nach Südafrika. Anlässlich seiner ersten Reise nach Südafrika im Jahr 1994 kündigte Bundesrat Cotti ein Entwicklungshilfeprogramm im Umfang von 80 Millionen an, welches von 1995 bis 1999 dauern soll und dessen Gelder insbesondere zur Finanzierung von Stipendien, für Kleinkredite, für Verbesserungen der Infrastruktur in den Städten (Instandsetzung der Townships, Wasserversorgung, Ausbau der Abwasserentsorgung und Strassenbau in der Agglomeration von Port Elizabeth), zur Unterstuitzung der Demokratiebewegung und zur Förderung der Menschenrechte verwendet werden sollten. So wurden beispielsweise je eine Million Franken zur Unterstützung der «Wahrheitskommission» und für die Ausarbeitung einer neuen Verfassung ausbezahlt, ferner wurde 1994 die Organisation der Wahlen finanziell unterstützt. Die DEZA hat in Johannesburg ein Koordinationsbüro eingerichtet. Anlässlich seiner 1998 unternommenen Reise besuchte Flavio Cotti mehrere von der Schweiz unterstützte Projekte in Port Elizabeth. Er bestätigte, dass die Zusammenarbeit mit Südafrika zwischen 2000 und 2004 fortgesetzt werde, wobei künftig besonderes Gewicht auf die Entwicklung des Bildungswesens, auf die weitere Verbesserung des Lebensraums in Port Elizabeth sowie auf die Gewährung von Krediten für private Unternehmer gelegt werden soll.

\section{Elfenbeinküste/Ghana}

Von Ende September bis Anfang Oktober reiste Staatssekretär Franz Blankart an der Spitze einer gemischten Kommission, der rund 15 Vertreter der Schweizer Wirtschaft angehörten, nach Abidjan und Accra. In Elfenbeinküste wurde eine Vereinbarung zur Zusammenarbeit im Bereich der Umschuldung und des Schuldendienstes unterzeichnet. Mit den Behörden Ghanas und einem Vertreter der multilateralen Agenturen wurde ein Programm zur technischen Unterstützung in Zusammenarbeit mit der UNCTAD, der IHK und der WTO vereinbart, welches die Teilnahme Ghanas an den Tätigkeiten der WTO erleichtern und die Integration des Landes in das multilaterale Handelssystem vorantreiben sollte. 


\section{QUELLEN}

«Bericht zur Aussenwirtschaftspolitik 97/1+2 und Botschaften zu Wirtschaftsvereinbarungen und zu Änderungen der Schweizer WTO-Verpflichtungsliste» vom Januar 1998, Bern (in Bundesblatt, Nr. 9, 10. März 1998, S. 759-1111, Bericht Nr. 97.090).

Communiqués der Informationsdienste der Bundesverwaltung, EDA, 27. Juli 1998 «Offizieller Besuch von Bundespräsident Flavio Cotti in Südafrika».

Pressemitteilungen des EDA, 3. März 1998 «Schweizer Wirtschaftsdelegation besucht Iran», 6. März 1998 «Tagung der gemischten Kommission Schweiz-Usbekistan», 25. Sepbember 1998 «Mission économique du Conseiller fédéral Pascal Couchepin au Mexique», 29. Oktober 1998 «Visite d'une délégation économique au Koweït et dans les Emirats Arabes Unis».

Flavio Cotti, «Wechselwirkungen zwischen der schweizerischen Aussenpolitik und Wirtschaftspolitik» in Schweiz. Zeitschrift für Volkswirtschaft und Statistik, 1996, Vol. 132 (3), S. 293-298.

Philippe Gugler, «Les défis pour la politique économique extérieure suisse dans le nouveau contexte économique mondial» in Schweiz. Zeitschrift für Volkswirtschaft und Statistik, 1996, Vol. 132 (3), S. 299-316.

«L'évolution des investissements directs en 1996», in Monnaie et conjoncture, Banque nationale suisse, Bulletin trimestriel No 4, décembre 1997.

La Liberté, 28. Juli 1998 (Flavio Cotti in Südafrika und Pascal Couchepin in Thailand), 20. November 1998 (Flavio Cotti in Indien).

Neue Zürcher Zeitung, 30. Januar 1998 «Ein Gespräch mit dem brasilianischen Präsidenten Cardoso», 3. August 1998 «Ausbau der Beziehungen zu Südafrika», 4. August 1998 «Bundesrat Couchepin in Thailand», 5. August 1998 «Cotti: Wir haben Vertrauen in Südafrika», 7. August 1998 «Malaysia umwirbt Schweizer Investoren», 5. November 1998 «Investitionsschutzabkommen mit Golf-Emiraten», 12. Oktober 1998 «Bundesrat Couchepin als Türöffner in Mexiko», 23. November 1998 «Staatsbesuch von Bundespäsident Cotti in Indien», 25. November 1998 «Dichtes Gesprächsprogramm in Dehli. Abschluss des Cotti-Besuchs».

Le Temps, 18. Juni 1998 «Afrique du Sud: un dossier brûlant», 20. Juli 1998 «Rouvrir le dossier Suisse-Afrique du Sud ne répond à aucune nécessité», 5. August 1998 «La Suisse veut renforcer ses liens économiques avec l'Afrique du Sud», 12. Oktober 1998 «Le Mexique demeure attractif pour les Suisses».

\section{INTERNET-ADRESSEN}

Eidgenössisches Departement für auswärtige Angelegenheiten:

Bundesamt für Aussenwirtschaft: http://www.admin.ch/bawi/index.html 